Volume 23 (2018) 157-166

DOI: $10.24330 /$ ieja.373659

\title{
SOME GRAPH ON PRIME IDEALS OF A COMMUTATIVE RING
}

\author{
Alpesh M. Dhorajia \\ Received: 20 April 2017; Revised: 15 July 2017; Accepted: 16 November 2017 \\ Communicated by Abdullah Harmancı
}

\begin{abstract}
Let $R$ be a commutative ring with an identity. Let $\operatorname{Spec}(R)$ be the set of all prime ideals of $R$ and $\operatorname{Max}(R)$ be the set of all maximal ideals of $R$. Let $S \subseteq \operatorname{Max}(R)$. We define an $S$-proper ideal sum graph on $\operatorname{Spec}(R)$, denoted by $\Gamma_{S}(\operatorname{Spec}(R), S)$, as an undirected graph whose vertex set is the set $\operatorname{Spec}(R)$ and, for two distinct vertices $P$ and $Q$, there is an arc from $P$ to $Q$, whenever $P+Q \subseteq \mathcal{M}$, for some maximal ideal $\mathcal{M}$ in $S$. In this paper, we prove that the complement graph of a proper sum graph $\Gamma(\operatorname{Spec}(R), S)$ is complete if and only if $R$ is an Artinian ring. We also study some basic properties of the graph $\Gamma_{S}(\operatorname{Spec}(R), S)$ such as connectivity, girth and clique number. We explore the influence of the ring theoretic properties of a commutative ring $R$ on the proper sum graph of $R$ and vice versa.
\end{abstract}

Mathematics Subject Classification (2010): 05C69, 13E05, 16P20

Keywords: Proper sum graph, Artinian ring, commutative ring

\section{Introduction}

Recently, the study of graphs associated to algebraic structures is a growing area of research. In 1988, Istvan Beck proposed the study of commutative rings by representing them as graphs [6]. He defined the zero-divisor graph for a ring to be the graph consisting of a vertex for each element in the ring, and an edge between two vertices $v$ and $w$ if $v w$ is equal to zero. These zero-divisor graphs marked the beginning of an approach to studying commutative rings with graphs. The zerodivisor graph studied extensively by many authors (see [1],[2],[4],[7],[12],[16],[17]). Many other classes of graphs associated to algebraic structures have also been actively investigated by many researchers (see [3], [10], [11], [13]).

Many of the algebraic properties are studied in terms of ideals of the rings, it is interesting to associate graph structure to the set of ideals and study the algebraic properties and the graph theoretical properties. To see some instances of these graphs, we refer to ([9], [14], [19]). In [15], Sharma and Bhatwadekar proposed a new approach that constructed another graph for the commutative ring $R$ known 
as a comaximal graph: the vertices are still elements of the ring, and there is an edge between two vertices $x$ and $y$ in $R$ if $x R+y R=R$. In [18], Ye and Wu defined the comaximal graphs on the set of ideals which are not contained in the Jacobson radical of the ring, two ideals are adjacent if and only if they are co-maximal.

In this paper, we define an $S$-proper sum graph on the set of prime ideals of a commutative ring. Let $R$ be a commutative ring with identity. By $\operatorname{Spec}(R)$ and $\operatorname{Max}(R)$, we denote the set of all prime ideals, and the set of all maximal ideals of $R$, respectively. Let $S$ be a nonempty subset of $\operatorname{Max}(R)$. We define the $S$-proper sum graph on the set of prime ideals of $R$, denoted by $\Gamma_{S}(\operatorname{Spec}(R), S)$, as an undirected graph whose vertex set is the set of all prime ideals and, for two distinct vertices $P$ and $Q$, there is an arc from $P$ to $Q$, denoted by $P \sim Q$, whenever $P+Q \subseteq \mathcal{M}$, for some maximal ideal $\mathcal{M}$ in $S$. In the case when $S=\operatorname{Max}(R)$, we denote the $S$-proper sum graph $\Gamma_{S}(\operatorname{Spec}(R), S)$ by $\Gamma(\operatorname{Spec}(R), S)$ and we simply call it the proper sum graph.

In Section 2, we study some basic properties of the graph $\Gamma_{S}(\operatorname{Spec}(R), S)$ such as connectivity, girth and clique number. In Section 3, we study the proper sum graph of product rings and investigate the relationship between the complement graph of proper sum graph and product rings. Throughout this paper, all the rings are assumed to be commutative with nonzero identity. By $\operatorname{Spec}(R)$ and $\operatorname{Max}(R)$, we denote the set of all prime ideals, and the set of all maximal ideals of $R$, respectively. Moreover, $V(I)$ is the set of all prime ideals of $R$ containing ideal $I$. We refer the standard terminology of commutative algebra following [5].

Now, we recall some definitions and notations on graphs. We use the standard terminology of graphs following [8]. Let $G=(V, E)$ be a graph, where $V$ is the set of vertices and $E$ is the set of edges. The graph $H=\left(V_{0}, E_{0}\right)$ is a subgraph of $G$ if $V_{0} \subseteq V$ and $E_{0} \subseteq E$. The distance between two distinct vertices $v_{1}$ and $v_{2}$ in $G$, denoted by $d\left(v_{1}, v_{2}\right)$, is the length of the shortest path connecting $v_{1}$ and $v_{2}$, if such a path exists; otherwise, we set $d\left(v_{1}, v_{2}\right):=\infty$. The diameter of a graph $G$ is $\operatorname{diam}(G)=\operatorname{Sup}\left\{d\left(v_{1}, v_{2}\right): v_{1}\right.$ and $v_{2}$ are distinct vertices of $\left.G\right\}$. The girth of $G$ is the length of the shortest cycle in $G$, denoted by $\operatorname{gr}(G)(\operatorname{gr}(G):=\infty$ if $G$ has no cycles). Also, for two distinct vertices $v_{1}$ and $v_{2}$ in $G$, the notation $v_{1} \sim v_{2}$ means that $v_{1}$ and $v_{2}$ are adjacent. A graph $G$ is said to be connected if there exists a path between any two distinct vertices, and it is complete if it is connected with diameter one. For a vertex $v$ in $G$, the degree of $v$ is the number of vertices adjacent to $v$ and it is denoted by $\operatorname{deg}(v)$. A vertex $v$ is an isolated vertex if $\operatorname{deg}(v)=0$. A clique of a graph is a complete subgraph of it and the number of vertices in a largest clique of $G$ is called the clique number of $G$ and is denoted by $\omega(G)$. An 
independent set of $G$ is a subset of the vertices of $G$ such that no two vertices in the subset represent an edge of $G$. The independence number of $G$, denoted by $\alpha(G)$, is the cardinality of a largest independent set. A nonempty subset $S$ of vertices of $G$ is a dominating set if every vertex in $V \backslash S$ is adjacent to some vertex in $S$. The domination number denoted by $\gamma(G)$ is defined to be the number of vertices in a smallest dominating set in $G$. A smallest dominating set is referred to as $\gamma$-set. For a positive integer $r$, an $r$-partite graph is one whose vertex set can be partitioned into $r$ subsets, so that no edge has both ends in any one subset.

\section{Basic properties of proper sum graph}

Throughout this paper $R$ is a commutative ring with nonzero identity and $S$ is a subset of $\operatorname{Max}(R)$. In this section, we study some basic properties of the graphs $\Gamma_{S}(\operatorname{Spec}(R), S)$. We begin this section with the following definition.

Definition 2.1. A set $S$ is said to be minimal with respect to intersection and we denote it by m.r.i., if for any two $m_{1}, m_{2} \in S$, the intersection $m_{1} \cap m_{2}$ does not contain any nontrivial prime ideal.

Example 2.2. Let $R=\mathbb{Z}$, then $\operatorname{Spec}(R)=\{(0),(p)$, where $p$ is prime $\}$. Let $S=\operatorname{Max}(R)$. Then clearly $S$ is minimal with respect to intersection.

Example 2.3. Let $R$ be any principal ideal domain which is not field and let $S=\operatorname{Max}(R)$. Then $S$ is minimal with respect to intersection.

Remark 2.4. If $R$ is a commutative ring with nonzero identity, then $\operatorname{Max}(R) \neq \emptyset$. Moreover, for any nonzero proper ideal $I$, there exists a maximal ideal $\mathcal{M}$ such that $I \subseteq \mathcal{M}$

Proposition 2.5. If the $S$-proper sum graph $\Gamma_{S}(\operatorname{Spec}(R), S)$ is connected and $S$ is m.r.i., then $R$ is a domain or a local ring.

Proof. If (0) is a prime ideal then clearly $R$ is an integral domain. If (0) is not a prime ideal, we show that $R$ is a local ring. Let $\mathcal{M}_{1}, \mathcal{M}_{2}$ be any two maximal ideals of $R$, we show that $\mathcal{M}_{1}=\mathcal{M}_{2}$. Since the graph $\Gamma_{S}(\operatorname{Spec}(R), S)$ is connected, we have a path joining $\mathcal{M}_{1}$ and $\mathcal{M}_{2}$, say $\mathcal{M}_{1} \sim P_{1} \sim \cdots \sim P_{n} \sim \mathcal{M}_{2}$. Note that all the prime ideals $P_{i}$ are nontrivial, for $1 \leq i \leq n$. Since $\mathcal{M}_{1}$ is adjacent to $P_{1}$, and $P_{1}$ is adjacent to say $P_{2}$, there exists an ideal $\mathcal{M} \in S$ such that $P_{1}+P_{2} \subseteq \mathcal{M}$. Clearly, $\mathcal{M}_{1} \cap \mathcal{M}$ contains a prime ideal $P_{1}$. Since $S$ is minimal with respect to intersection, we have $P_{1}=(0)$ or $\mathcal{M}_{1}=\mathcal{M}$. By the similar arguments, we have $\mathcal{M}_{1}=\mathcal{M}_{2}$. Therefore $R$ is a local ring. 
Proposition 2.6. If $R$ is an integral domain or a local ring, then the proper sum graph $\Gamma(\operatorname{Spec}(R), S)$ is connected.

Proof. If $R$ is a domain, then (0) is a prime ideal. Therefore for any two nontrivial prime ideals $P$ and $Q$, we have a path joining $P$ to $Q$ is given by $P \sim(0) \sim Q$. If $R$ is a local ring with a maximal ideal $\mathcal{M}$, then for any two prime ideals $P$ and $Q, P+Q \subseteq \mathcal{M}$. Therefore $P \sim Q$. Hence the proper sum graph $\Gamma(\operatorname{Spec}(R), S)$ is connected.

Corollary 2.7. If $R$ is an integral domain or a local ring, then $\operatorname{diam}(\Gamma(\operatorname{Spec}(R), S)) \leq$ 2 .

Theorem 2.8. The proper sum graph $\Gamma(\operatorname{Spec}(R), S)$ is complete if and only if $R$ is a local ring.

Proof. Suppose that $R$ is not a local ring. Let $\mathcal{M}_{1}$ and $\mathcal{M}_{2}$ be any two distinct maximal ideals of $R$. Since $\Gamma(\operatorname{Spec}(R), S)$ is complete, we have $\mathcal{M}_{1} \sim \mathcal{M}_{2}$. This is a contradiction, as $\mathcal{M}_{1}+\mathcal{M}_{2}=R$, hence our assumption is wrong, $R$ is a local ring. Conversely, Since $R$ is a local ring with maximal ideal $\mathcal{M}$, for any two prime ideals $P$ and $Q, P+Q \subseteq \mathcal{M}$. Therefore $P \sim Q$, hence the graph is complete.

Theorem 2.9. If $R$ is a principal ideal domain, then $\Gamma(\operatorname{Spec}(R), S)$ is a star graph. The converse holds if $R$ is a unique factorization domain.

Proof. Since $R$ is a domain, (0) is a prime ideal. As $R$ is a principal ideal domain, every nontrivial prime ideals are maximal. Therefore we have $(0) \sim P$ for all nontrivial prime ideal $P$. Also any two nontrivial prime ideals are co-maximal, hence they are not adjacent. Hence $\Gamma(\operatorname{Spec}(R), S)$ is a star graph. Now suppose that $\Gamma(\operatorname{Spec}(R), S)$ is a star graph and $R$ is a unique factorization domain. Since the graph $\Gamma(\operatorname{Spec}(R), S)$ is a star graph, all nontrivial prime ideals are maximal. It is enough to show that every nonzero prime ideal is generated by one element. Suppose a prime ideal $P$ is minimally generated by two elements say $x$ and $y$. Since $R$ is a UFD, we can assume that $x$ and $y$ are irreducible elements. Clearly $(x)$ is a nonzero prime ideal of $R$ and hence there is a chain $(0) \sim(x) \sim(x, y) \sim(0)$ in $\Gamma(\operatorname{Spec}(R), S)$. Contradiction as $\Gamma(\operatorname{Spec}(R), S)$ is a star graph and hence every nonzero prime ideal generated by one element.

Theorem 2.10. Let $R$ be a non local ring. If the proper sum graph $\Gamma_{S}(\operatorname{Spec}(R), S)$ is a star graph, with $S$ is m.r.i., then $R$ is an integral domain of dimension $\leq 1$.

Proof. Let $P$ be a vertex in a graph such that $P \sim Q$ for all $Q \in \operatorname{Spec}(R)$. First, we show that $(0)$ is a prime ideal. Let $\mathcal{M}_{1}, \mathcal{M}_{2}$ be two maximal ideals such 
that $P \sim \mathcal{M}_{1}$ and $P \sim \mathcal{M}_{2}$. As $P+\mathcal{M}_{1}=\mathcal{M}_{1}$ and $P+\mathcal{M}_{2}=\mathcal{M}_{2}$, we have $P \subseteq \mathcal{M}_{1} \cap \mathcal{M}_{2}$. Since $S$ is minimal with respect to intersection, $P=(0)$. Therefore (0) is a prime ideal and hence $R$ is a domain. Suppose $\operatorname{dim} R \geq 2$. There exists a chain of prime ideals of length at least two as follows:

$$
P_{0} \subsetneq P_{1} \subsetneq P_{2} .
$$

Clearly, $P_{0}=(0)$. Since $\Gamma_{S}(\operatorname{Spec}(R), S)$ is a star graph, $P_{0} \sim P_{1}$, i.e. there is a maximal ideal $\mathcal{M}$ such that $P_{0}+P_{1} \subseteq \mathcal{M}$. If $P_{1} \neq \mathcal{M}$, then we have a cycle $P_{0} \sim P_{1} \sim \mathcal{M} \sim \cdots \sim P_{0}$, which is a contradiction as $\Gamma_{S}(\operatorname{Spec}(R), S)$ is a star graph. Suppose $P_{1}=\mathcal{M}$. Since $\mathcal{M}$ is a maximal ideal, $P_{1} \subsetneq P_{2}$ is not possible. Hence there does not exist any chain of prime ideals of length two or more. Therefore $\operatorname{dim} R \leq 1$.

Proposition 2.11. If the proper sum graph $\Gamma_{S}(\operatorname{Spec}(R), S)$ is a star graph, then $S=\operatorname{Max}(R)$.

Proof. Let $P$ be a fix vertex such that $P \sim Q$ for all vertex $Q$. Let $\mathcal{M}$ be any maximal ideal of $R$. Since $P \sim \mathcal{M}$, we have $\mathcal{M} \in S$.

Proposition 2.12. If $R$ is ring of dimension $\geq 2$, then $\operatorname{gr}(\Gamma(\operatorname{Spec}(R), S)=3$.

Proof. Since $\operatorname{dim} R \geq 2$, there exists a chain of prime ideals such that $P_{0} \subsetneq P_{1} \subsetneq$ $P_{2}$. Since $R$ is a commutative ring with identity, there exists a maximal ideal $\mathcal{M}$ such that $P_{2} \subseteq \mathcal{M}$. Therefore, we have the cycle $P_{0} \sim P_{1} \sim \mathcal{M} \sim P_{0}$.

Proposition 2.13. If $\operatorname{dim} R=1$ and the proper sum $\operatorname{graph} \Gamma(\operatorname{Spec}(R), S)$ has a cycle, then $R$ is not an integral domain.

Proof. We show that (0) is not a prime ideal. By contradiction, assume that (0) is a prime ideal. Let $P_{0} \sim P_{1} \sim P_{2} \sim P_{0}$ be a cycle in $\Gamma(\operatorname{Spec}(R), S)$. There exists a maximal ideal $\mathcal{M} \in S$ such that $P_{0}+P_{1} \subseteq \mathcal{M}$. Either $P_{0}$ or $P_{1}$ is a nontrivial prime ideal, say $P_{0}$ is nontrivial. Since (0) is a prime ideal and $\operatorname{dim} R=1, P_{1}$ is a maximal ideal. As $P_{0}$ and $P_{1}$ are adjacent, $P_{0}+P_{1} \subseteq \mathcal{M}$ and hence $P_{1}=\mathcal{M}$. Since $\operatorname{dim} R=1$ and $P_{2}$ is a nontrivial prime ideal, $P_{2}$ is also a maximal ideal. Therefore $P_{1}$ and $P_{2}$ are co-maximal, which is not possible as $P_{1} \sim P_{2}$. Hence (0) is not a prime ideal and $R$ is not an integral domain.

Proposition 2.14. Let $R$ be a ring such that $|\operatorname{Min}(R)|<\infty$. Then the following statements hold:

(a) The $\operatorname{Spec}(R)$ is connected if and only if $\Gamma(\operatorname{Spec}(R), S)$ is connected and $\operatorname{diam}(\Gamma(\operatorname{Spec}(R), S)) \leq 2|\operatorname{Min}(R)|$. 
(b) $\gamma(\Gamma(\operatorname{Spec}(R), S)) \leq|\operatorname{Min}(R)|$.

Proof. (a) By contradiction, suppose $\operatorname{Spec}(R)$ is not connected. There exist two nonempty sets $V(I)$ and $V(J)$ such that $V(I) \cup V(J)=\operatorname{Spec}(R)$ and $V(I) \cap V(J)=$ $\emptyset$ for some ideals $I$ and $J$. Let $P \in V(I)$ and $Q \in V(J)$. Since $\Gamma(\operatorname{Spec}(R), S)$ is connected, there is a path as follows:

$$
P \sim P_{1} \sim P_{2} \sim \cdots P_{n} \sim Q
$$

Since $P \sim P_{1}$, there exists a maximal ideal $\mathcal{M}$ such that $P+P_{1} \subset \mathcal{M}$. Since $P \in V(I), \mathcal{M} \in V(I)$ and hence $P_{2} \in V(I)$. Applying the similar arguments, we can show that $P_{n} \in V(I)$. Since $P_{n} \sim Q$ there exists a maximal ideal $\mathcal{N}$ such that $P_{n}+Q \subset \mathcal{N}$. Since $P_{n} \subset \mathcal{N}, \mathcal{N} \in V(I)$ also as $Q \subset \mathcal{N}, \mathcal{N} \in V(J)$, contradiction as $V(I) \cap V(J)=\emptyset$.

Let $\operatorname{Min}(R)=\left\{P_{1}, P_{2}, \ldots, P_{r}\right\}$. First suppose $r=2$. Let $P$ and $Q$ be any two prime ideals of $R$. We show that there is a path of length at most four joining $P$ to $Q$. There exist minimal prime ideals $P_{1}$ and $Q_{1}$ such that $P_{1} \subseteq P$ and $Q_{1} \subseteq Q$. Since $\operatorname{Spec}(R)$ is connected, we have $V\left(P_{1}\right) \cap V\left(Q_{1}\right) \neq \emptyset$. There exists a prime ideal $P_{2} \in V\left(P_{1}\right) \cap V\left(Q_{1}\right)$. Since $R$ is a commutative ring with identity, we have a path $P \sim P_{1} \sim P_{2} \sim Q_{1} \sim Q$. Now, suppose $r>2$. Let $1 \leq i \leq l_{1}$ such that $P \in V\left(P_{i}\right)$, for $l_{1}<i \leq l_{2}$ such that $Q \in V\left(P_{i}\right)$ and $l_{2}<i \leq r$ such that $P, Q \notin V\left(P_{i}\right)$. Since $\operatorname{Spec}(R)$ is connected, there exist $1 \leq i_{1} \leq \cdots \leq i_{k} \leq$ $r$ such that $V\left(P_{i_{l}}\right) \cap V\left(P_{i_{l+1}}\right) \neq \emptyset$ for $1 \leq l \leq k$ and $\left(\bigcup_{i=1}^{l_{1}} P_{i}\right) \cap V\left(P_{i_{1}}\right) \neq \emptyset$, $\left(\bigcup_{i=l_{1}+1}^{l_{2}} P_{i}\right) \cap V\left(P_{i_{k}}\right) \neq \emptyset$. Therefore, it is easy to see that there is a path between $P$ and $Q$ of length at most $2|\operatorname{Min}(R)|$.

(b) Let $S=\left\{P_{1}, P_{2}, \ldots, P_{n}\right\}$ be a dominating set in $\Gamma(\operatorname{Spec}(R), S)$. Therefore, for any $Q \in \operatorname{Spec}(R) \backslash S$ there is a $P_{i} \in S$ such that $Q \sim P_{i}$ for some $1 \leq i \leq n$. Let $Q_{1}, Q_{2}, \ldots, Q_{r}$ be the distinct minimal prime ideals of $R$ such that each prime ideal $P_{i}$ contains at least one $Q_{j}$ for $1 \leq j \leq r$. Therefore $r \leq n$. We show that $T=\left\{Q_{1}, Q_{2}, \ldots, Q_{r}\right\}$ is also a dominating set. Let $P^{\prime}$ be any prime ideal which is not in $T$. Suppose $P^{\prime} \notin S$. Since $S$ is a dominating set, there exists a prime ideal $P_{i} \in S$ such that $P^{\prime} \sim P_{i}$. There exists a maximal ideal $\mathcal{M}$ such that $P^{\prime}+P_{i} \subseteq \mathcal{M}$. By construction of the set $T$, there exists a $Q_{j} \in T$ such that $Q_{j} \subseteq P_{i}$ for some $1 \leq j \leq r$. Therefore $P^{\prime}+Q_{j} \subseteq \mathcal{M}$, and hence $P^{\prime} \sim Q_{j}$. Therefore $T$ is a dominating set. Now suppose $P^{\prime} \in S$. There exists $Q_{j} \in T$ such that $Q_{j} \subseteq P^{\prime}$. Since $R$ is a commutative ring with unity, there exists a maximal ideal $\mathcal{M}_{1}$ such that $P^{\prime} \subseteq \mathcal{M}_{1}$. Therefore $P^{\prime} \sim Q_{j}$, hence $T$ is a dominating set. 


\section{Proper sum graph of direct product of rings}

In this section, we study the proper sum graph of product of rings $R_{i}$ for $1 \leq$ $i \leq n$. We relate the clique number, independent number and dominating number of $\Gamma(\operatorname{Spec}(R), S)$ and that of $\Gamma\left(\operatorname{Spec}\left(R_{i}\right), S_{i}\right)$ for $1 \leq i \leq n$.

Theorem 3.1. Let $R_{1}, R_{2}, \ldots, R_{n}$ be commutative rings with identities and $R=$ $R_{1} \times R_{2} \times \cdots \times R_{n}$. Then $\Gamma(\operatorname{Spec}(R), S)=\coprod_{i=1}^{n} \Gamma\left(\operatorname{Spec}\left(R_{i}\right), S_{i}\right)$, where $S_{i}=$ $\operatorname{Max}\left(R_{i}\right)$ for $1 \leq i \leq n$, where $\amalg$ denotes the disjoint union of sets.

Proof. Any prime ideal $P$ of $R$ is of the form $R_{1} \times R_{2} \times \cdots \times R_{i-1} \times P_{i} \times R_{i+1} \times$ $\cdots \times R_{n}$, where $P_{i}$ is a prime ideal in $R_{i}$ and any maximal ideal of $R$ is of the form $R_{1} \times R_{2} \times \cdots \times R_{i-1} \times \mathcal{M}_{i} \times R_{i+1} \times \cdots \times R_{n}$ for some maximal ideal $\mathcal{M}_{i}$ of $R_{i}$. Therefore, we can associate each prime ideal $P_{i}$ of $R_{i}$ to prime ideal $R_{1} \times$ $R_{2} \times \cdots \times R_{i-1} \times P_{i} \times R_{i+1} \times \cdots \times R_{n}$ of $R$. Clearly, if $P_{i}$ and $Q_{i}$ are adjacent in $\Gamma\left(\operatorname{Spec}\left(R_{i}\right), S_{i}\right)$, then we have $R_{1} \times R_{2} \times \cdots \times R_{i-1} \times P_{i} \times R_{i+1} \times \cdots \times R_{n}$ and $R_{1} \times R_{2} \times \cdots \times R_{i-1} \times Q_{i} \times R_{i+1} \times \cdots \times R_{n}$ are adjacent in $\Gamma(\operatorname{Spec}(R), S)$ and vice versa.

Corollary 3.2. If $R$ is an Artinian ring, then the proper sum graph $\Gamma(\operatorname{Spec}(R), S)$ is disconnected or it is an isolated vertex.

Corollary 3.3. If $R$ is an Artinian non local ring, then the complement graph of the proper sum graph $\Gamma(\operatorname{Spec}(R), S)$ is complete graph.

Corollary 3.4. Let $R_{1}, R_{2}, \ldots, R_{n}$ be commutative rings with identities and $R=$ $R_{1} \times R_{2} \times \cdots \times R_{n}$. The following statements hold:

(a) $\gamma(\Gamma(\operatorname{Spec}(R), S)) \leq\left|\operatorname{Min}\left(R_{1}\right)\right|+\left|\operatorname{Min}\left(R_{2}\right)\right|+\ldots+\left|\operatorname{Min}\left(R_{n}\right)\right|$.

(b) $\alpha(\Gamma(\operatorname{Spec}(R), S))=\left|\operatorname{Max}\left(R_{1}\right)\right|+\ldots+\left|\operatorname{Max}\left(R_{n}\right)\right|$.

(c) $\omega(\Gamma(\operatorname{Spec}(R), S))=\operatorname{Max}\left\{\omega\left(\Gamma\left(\operatorname{Spec}\left(R_{1}\right), S_{1}\right)\right), \ldots, \omega\left(\Gamma\left(\operatorname{Spec}\left(R_{n}\right), S_{n}\right)\right)\right\}$.

Theorem 3.5. Suppose $R$ is a reduced Noetherian ring. The complement graph of the proper sum graph $\Gamma(\operatorname{Spec}(R), S)$ is complete multipartite if and only if $R$ is a direct product of local rings.

Proof. Suppose the complement graph of $\Gamma(\operatorname{Spec}(R), S)$ is complete multipartite with the vertex-sets $V_{1}, V_{2}, \ldots, V_{n}$. Clearly, the set of vertices in $V_{i}$ forms a complete subgraph of $\Gamma(\operatorname{Spec}(R), S)$ for $1 \leq i \leq n$. Since the subgraph on $V_{i}$ is complete, $V_{i}$ has a maximal ideal. Also it is easy to see that $V_{i}$ has a unique maximal ideal, say $\mathcal{M}$. Now, consider a set $A$, consists of all prime ideals of $R$ which is contained in $\mathcal{M}$. We now show that $A=V_{i}$. Let $P$ be any prime ideal of $R$ such that $P \subseteq \mathcal{M}$. Clearly, 
$P \sim \mathcal{M}$. Therefore in the complement graph, $P$ and $\mathcal{M}$ are not adjacent. Suppose $P \in V_{j}$ for some $i \neq j$. Since the complement graph of $\Gamma(\operatorname{Spec}(R), S)$ is complete multipartite, $P \sim \mathcal{M}$ in complement graph. Since $P \sim \mathcal{M}$ in $\Gamma(\operatorname{Spec}(R), S)$, a contradiction. Hence $A=V_{i}$. Since $R$ is a Noetherian ring, $R$ has finitely many minimal prime ideals. Let $P_{1}, P_{2}, \ldots, P_{m}$ be the minimal prime ideals of $R$. Clearly, for each $i$, there exists at least one $j$ such that $P_{j} \in V_{i}$. Let $P_{i_{1}}, \ldots, P_{i_{r}}$ be minimal prime ideals such that $P_{i_{1}}, \ldots, P_{i_{r}} \in V_{i}$. Since $V\left(P_{i_{1}}\right) \cup V\left(P_{i_{2}}\right) \cup \cdots \cup V\left(P_{i_{r}}\right)=$ $V\left(P_{i_{1}} \cap P_{i_{2}} \cap \cdots \cap P_{i_{r}}\right)$, we can show that $V\left(P_{i_{1}} \cap P_{i_{2}} \cap \cdots \cap P_{i_{r}}\right)=V_{i}$. Let $P$ be any prime ideal of $R$ such that $P \in V_{i}$, i.e. $P \subseteq \mathcal{M}$. If $P$ is a minimal prime ideal of $R$, then clearly $P \in V\left(P_{i_{1}} \cap P_{i_{2}} \cap \cdots \cap P_{i_{r}}\right)$. Assume that $P$ is not a minimal prime ideal of $R$. Since $P$ is not a minimal prime ideal, there exists a minimal prime ideal of $R$, say $Q$ such that $Q \subsetneq P$. If $Q \in\left\{P_{i_{1}}, \ldots, P_{i_{r}}\right\}$, then clearly $P \in V\left(P_{i_{1}} \cap P_{i_{2}} \cap \cdots \cap P_{i_{r}}\right)$. Suppose $Q \notin\left\{P_{i_{1}}, \ldots, P_{i_{r}}\right\}$. As $Q \subseteq \mathcal{M}$ and $Q$ is minimal, hence $Q=P_{i_{k}}$ for some $1 \leq k \leq r$ which is a contradiction. Therefore $P \in V\left(P_{i_{1}} \cap P_{i_{2}} \cap \cdots \cap P_{i_{r}}\right)$. Let $P$ be any prime ideal containing $P_{i_{k}}$ for some $1 \leq k \leq r$. We show that $P \subseteq \mathcal{M}$ i.e. $P \sim \mathcal{M}$ in the graph $\Gamma(\operatorname{Spec}(R), S)$. Suppose $P$ is not adjacent to $\mathcal{M}$ in $\Gamma(\operatorname{Spec}(R), S)$. Since $R$ is a commutative ring with unity, there exists a maximal ideal say $\mathcal{M}_{1}$ of $R$ such that $P_{i_{r}} \subseteq P \subseteq \mathcal{M}_{1}$. Since $P \subseteq \mathcal{M}_{1}$ there is a path $\mathcal{M} \sim P_{i_{k}} \sim \mathcal{M}_{1}$ in $\Gamma(\operatorname{Spec}(R), S)$. As the complement graph of $\Gamma(\operatorname{Spec}(R), S)$ is complete multipartite, $\mathcal{M}, \mathcal{M}_{1}$ and $P_{i_{k}}$ belong to the same vertex set $V_{i}$. Since $V_{i}$ has a unique maximal ideal $\mathcal{M}$, a contradiction. Therefore $V\left(P_{i_{1}} \cap P_{i_{2}} \cap \cdots \cap P_{i_{r}}\right)=V_{i}$. Let $I_{i}=P_{i_{1}} \cap P_{i_{2}} \cap \cdots \cap P_{i_{r}}$ for $1 \leq i \leq n$. Then clearly $\operatorname{Spec}(R)$ is a disjoint union of finitely many closed subsets. It is easy to see that each of $I_{i}$ are co-maximal ideals of $R$. Since $R$ is a reduced ring, we have $R \cong R / I_{1} \times \cdots \times R / I_{n}$. Clearly, $R / I_{i}$ is a local ring.

Now conversely, by Theorem 3.1, the graph $\Gamma(\operatorname{Spec}(R), S)$ is a disjoint union of sub graphs $\Gamma\left(\operatorname{Spec}\left(R_{i}\right), S_{i}\right)$ for $1 \leq i \leq n$. Since each $R_{i}$ is a local ring, by Theorem 2.8 , each $\Gamma\left(\operatorname{Spec}\left(R_{i}\right), S_{i}\right)$ is a complete graph. Therefore, the complement graph of $\Gamma_{S}(\operatorname{Spec}(R), S)$ is complete multipartite.

Corollary 3.6. The complement graph of the proper sum graph $\Gamma(\operatorname{Spec}(R), S)$ is complete if and only if $R$ is an Artinian ring.

Acknowledgment. I sincerely thank the referee for going through the paper with great care. A detailed list of suggestions and corrections by the referee improved the exposition considerably. 


\section{References}

[1] S. Akbari and A. Mohammadian, On the zero-divisor graph of a commutative ring, J. Algebra, 274(2) (2004), 847-855.

[2] D. F. Anderson and A. Badawi, On the zero-divisor graph of a ring, Comm. Algebra, 36(8) (2008), 3073-3092.

[3] D. F. Anderson and A. Badawi, The total graph of a commutative ring, J. Algebra, 320(7) (2008), 2706-2719.

[4] D. F. Anderson and P. S. Livingston, The zero-divisor graph of a commutative ring, J. Algebra, 217(2) (1999), 434-447.

[5] M. F. Atiyah and I. G. MacDonald, Introduction to Commutative Algebra, Addison-Wesley Publishing Co., Reading, Mass.-London-Don Mills, Ont., 1969.

[6] I. Beck, Coloring of commutative rings, J. Algebra, 116(1) (1988), 208-226.

[7] N. Bloomfield and C. Wickham, Local rings with genus two zero divisor graph, Comm. Algebra, 38(8) (2010), 2965-2980.

[8] J. A. Bondy and U. S. R. Murty, Graph Theory with Applications, American Elsevier Publishing Co., Inc., New York, 1976.

[9] I. Chakrabarty, S. Ghosh, T. K. Mukherjee and M. K. Sen, Intersection graphs of ideals of rings, Discrete Math., 309(17) (2009), 5381-5392.

[10] A. M. Dhorajia, Total graph of the ring $\mathbb{Z}_{n} \times \mathbb{Z}_{m}$, Discrete Math. Algorithms Appl., 7(1) (2015), 1550004 (9 pp).

[11] A. M. Dhorajia and J. M. Morzaria, Domination in total graphs of small rings, Discrete Math. Algorithms Appl., 8(4) (2016), 1650069 (11 pp).

[12] H. R. Maimani, M. R. Pournaki and S. Yassemi, Zero-divisor graph with respect to an ideal, Comm. Algebra, 34(3) (2006), 923-929.

[13] H. R. Maimani, C. Wickham and S. Yassemi, Rings whose total graphs have genus at most one, Rocky Mountain J. Math., 42(5) (2012), 1551-1560.

[14] M. J. Nikmehr and F. Shaveisi, The regular digraph of ideals of a commutative ring, Acta Math. Hungar., 134(4) (2012), 516-528.

[15] P. K. Sharma and S. M. Bhatwadekar, A note on graphical representation of rings, J. Algebra, 176(1) (1995), 124-127.

[16] S. Spiroff and C. Wickham, A zero divisor graph determined by equivalence classes of zero divisors, Comm. Algebra, 39(7) (2011), 2338-2348.

[17] C. Wickham, Classification of rings with genus one zero-divisor graphs, Comm. Algebra, 36(2) (2008), 325-345. 
[18] M. Ye and T. Wu, Co-maximal ideal graphs of commutative rings, J. Algebra Appl., 11(6) (2012), 1250114 (14 pp).

[19] M. Ye, T. Wu, Q. Liu and J. Guo, Graph properties of co-maximal ideal graphs of commutative rings, J. Algebra Appl., 14(3) (2015), 1550027 (13 pp).

\author{
Alpesh M. Dhorajia \\ Department of Mathematics \\ Birla Institute of Technology and Science \\ Pilani, India \\ e-mail: alpesh@goa.bits-pilani.ac.in
}

